\title{
Successful application of World Health Organization multimodal strategy for hand hygiene
}

\author{
JM Alsalman*, Jameela Alsalman, Fatima Isa, Bahija Ahmed, Zaineb Alrayees, Khadmiya Altitoon, \\ Ameena Al Sayegh, Najat Salem \\ From International Conference on Prevention \& Infection Control (ICPIC 2011) \\ Geneva, Switzerland. 29 June - 2 July 2011
}

\section{Introduction / objectives}

Hand hygiene is an effective means of preventing hospital-associated infection (HAI). Compliance among health care workers( $\mathrm{HCWs}$ ) is the main strategic goal for the infection control committee as per the Ministry of Health collaboration with World Health Organization's (WHO) Global Patient Safety Challenge since October 2005.

\section{Methods}

The following steps were taken by the infection control team in A secondary/tertiary care hospital (>1000 bed) and in a psychiatry hospital ( $>230$ beds), for the last 3 years; were we:

- Acknowledged the importance of HAI and hand hygiene and started the surveillance.

- Developed ongoing campaigns at national or subnational levels to promote and improve hand hygiene among HCWs; where we ran two campaigns per year

- Made information available on HAI and hand hygiene for all HCWs through educational sessions, Â materials, newsletter, workshops and a designed website.

- Shared experiences with the WHO

- Used the WHO strategies, guidelines and tools to tackle HAI

- Promoted the highest standards of practice and behavior to reduce HAI;

- Encouraged the senior management support for implementation of interventions to reduce HAIs.
- Created a voluntary team of medical residents to participate in the audit.

\section{Results}

HCWs compliance with hand hygiene recommendations was low during the first Audit less than 10\% (2007), with differences between doctors and nurses. Several activities introduced for HCWs during the whole year and on $5^{\text {th }}$ of each month. A group developed a hospital campaigns using the WHO multimodal strategy and observational tools. Opportunities for hand hygiene were audited during three years period. The compliance were reviewed which showed a significant increase in the compliance above $70 \%$ in 2010 .

\section{Conclusion}

The application of WHO multimodal strategy for hand hygiene helped in increasing the compliance of HCWs with hand hygiene, aiming to reach $100 \%$.

\section{Disclosure of interest}

None declared.

Published: 29 June 2011

doi:10.1186/1753-6561-5-S6-P256

Cite this article as: Alsalman et al:: Successful application of World

Health Organization multimodal strategy for hand hygiene. BMC

Proceedings 2011 5(Suppl 6):P256. 